\title{
CHARTS FOR CONFIDENCE LIMITS ON THE MULTIPLE CORRELATION COEFFICIENT
}

\author{
AKIRA NUSHI AND SUKEYORI SHIBA ${ }^{1,2}$ \\ Depariment of Educational Psychology, Lniversity of Tokyo
}

\begin{abstract}
The confidence intervals on the multiple correlation coefficient were computed by using directly Fisher's formula (1928) for several sets of variables including both cases of even and odd numbers for each of the values $\mathcal{N}$ (sample size $)=15,20,30,40,50,75,100$ and 200 . These figures can be used not only to estimate the 90 percent confidence interval but to test hypothesis $\mathrm{H}_{0}: \rho=\rho_{0}$, where $\rho_{0}$ is not necessarily zero.
\end{abstract}

Fisher (1928) derived the density of the square of the sample multiple correlation coefficient, $r^{2}$. between a variable $X_{1}$ and a set of variables $\left(\mathrm{X}_{2}, \mathrm{X}_{3}, \ldots, \mathrm{X}_{p}\right)$ based on a sample of $V=n+1$, assuming that the sample had been randomly drawn from a $p$-dimensional multivariate normal population. A formula for the density is expressed by the sum of infinite series on integer $:$ :

$$
\begin{aligned}
& \frac{\left(1-r^{2}\right)^{\frac{1}{2} ; n-p^{p-1}}\left(1-\rho^{2}\right)^{\frac{1}{2} n}}{\Gamma\left[\frac{1}{2}(n-p+1)\right] \Gamma\left(\frac{1}{2} n\right)} \\
& \quad \times \sum_{\mu=0}^{\infty} \frac{\left(o^{2}\right)^{\mu}\left(r^{2}\right)^{\frac{1}{2}(p-1)+u-1} \Gamma^{2}\left(\frac{1}{2} n+\mu\right)}{n ! \Gamma\left[\frac{1}{2}(p-1)+\mu\right]}
\end{aligned}
$$

where $\rho$ is the corresponding population multiple correlation coefficient (Anderson, 1958). The density is also expressed in other forms (Nabeya, 1948).

Any of them requires much computational work and attempt to get confidence intervals for estimation of population multiple correlation coefficient has been almost impossible until the advent of the high-speed computer. Charts prepared by Ezekiel and Fox (1963), and tables

1 The authors are indebted to Dr. Takeo Nakano of Rikkyo University who gave many suggestions.

2 This study was supported by a Grant in Aid for Scientific Researches, Ministry of Education. prepared by Kramer (1963) were available for finding the confidence intervals. However, the use of these charts and tables was limited for the cases when $p-1$ is even.

Directly from the formula (1), we computed confidence intervals when $p-1$ 's are $2,3,4,5$, $6,7,8$ and 10 which include the case of odd numbers, under the condition that the errors are sufficiently less than required in drawing our charts. ${ }^{3,4}$ We, unfortunately, cannot give any simple description on the amount of errors in our computations since it depends on the various combinations of parameter values.

Figs. 1, 2, 3, 4, 5, 6, 7 and 8 show left and right $5.0^{\circ}$ tail points of distribution of sample multiple correlation for given population values for the sample sizes $\mathcal{N}$ which are indicated on each curve. Some curves for lower limits are omitted to avoid visual complexity. These figures can be used to estimate the confidence interval with confidence coefficient 0.90 . For example, suppose we have obtained sample multiple correlation coefficient $r=0.50$ between $\mathrm{X}_{1}$ and $\left(\mathrm{X}_{2}, \mathrm{X}_{3}, \mathrm{X}_{4}\right)(p-1=3)$ from the sample of 100 observations $(N=100)$. Turning to Fig. 2, we note that the ordinate at $r=0.50$

a All results were obtained on the HITAC 8800 , 8700 at the University of Tokyo Computer Centre.

4 Our program contains function subprogram of logarithmic gamma function $\ln \Gamma(\mathrm{x})$ which is appeared in "Statistical tables and formulas with computer applications" (Yamauchi, 1972). 

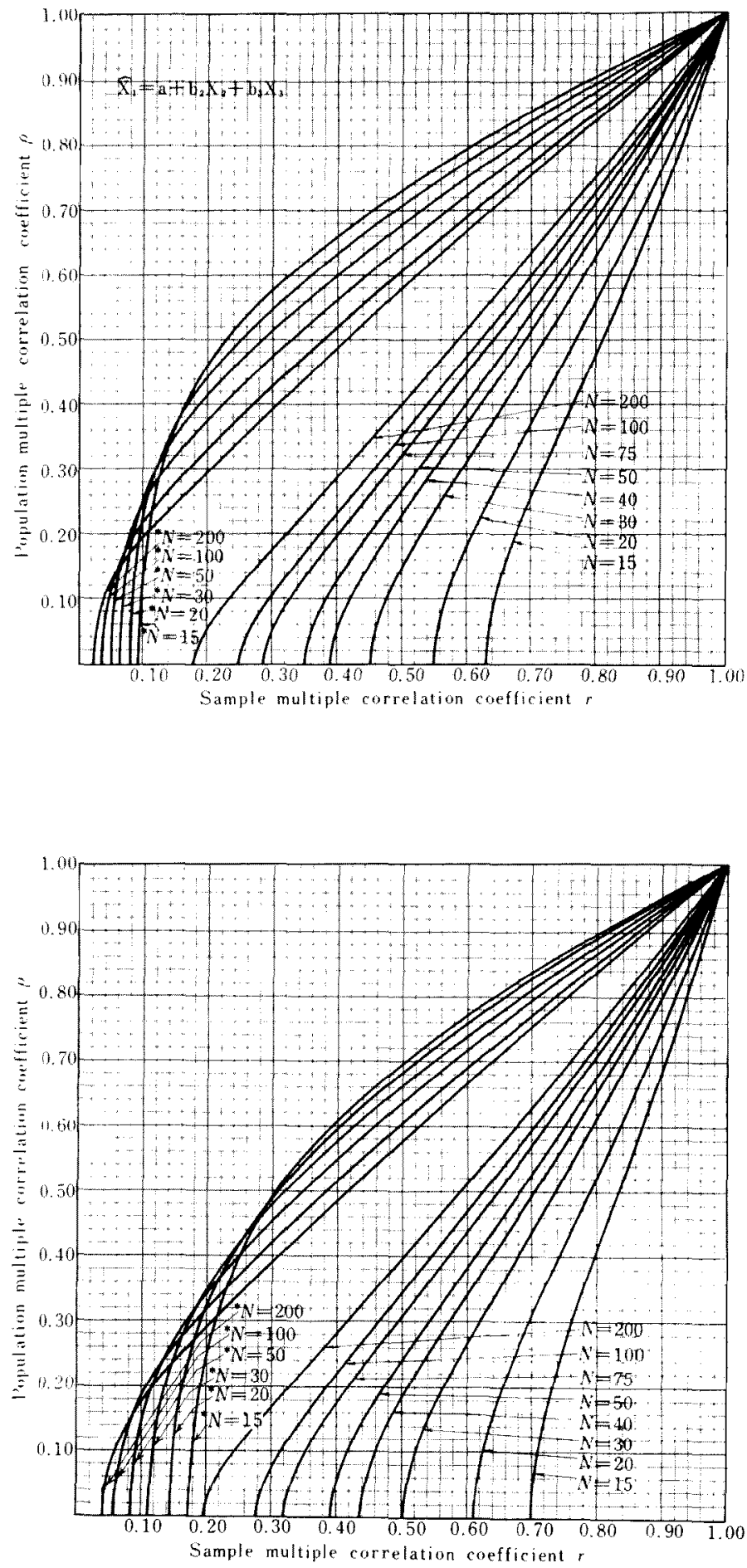

Fig. 1. Curves of left and right $5^{\circ}$ in tail points distribution of sample multiple correlation coefficient for the different sample sizes with 2 independent variables. (* Curves for the lower tails)
FIG. 2. Curves of left and right $5 \%$ tail points of distribution of sample multiple correlation coefficient for the different sample sizes with 3 independent variables. (* Curves for the lower tails) 
Fig. 3. Curves of left and right $5^{\circ}$ tail points of distribution of sample multiple correlation coefficient for the different sample sizes with 4 independent variables. (* Curves for the lower tails)
FIG. 4. Curves of left and right $5 \%$ tail points of distribution of sample multiple correlation coefficient for the different sample sizes with 5 independent variables. (* Curves for the lower tails)
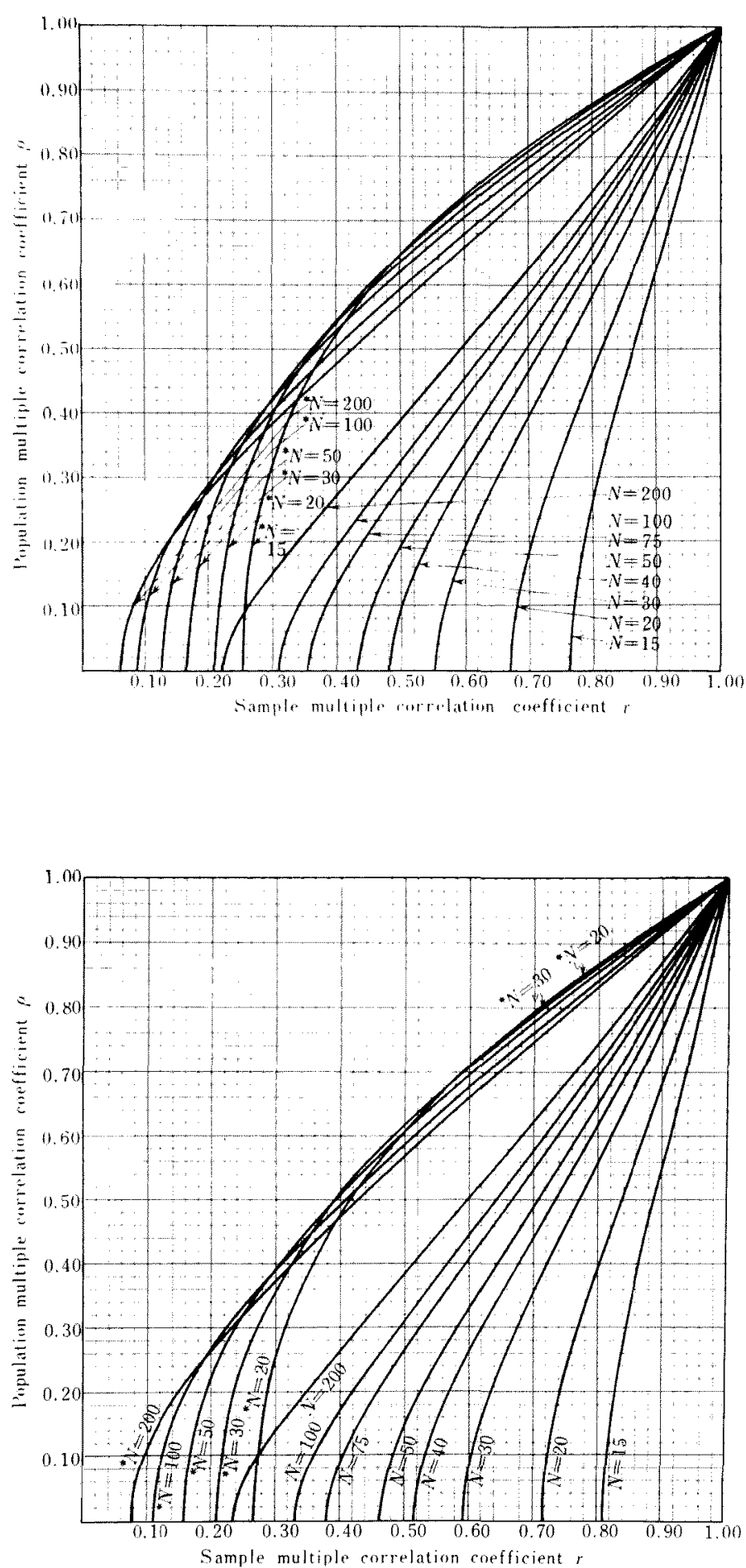

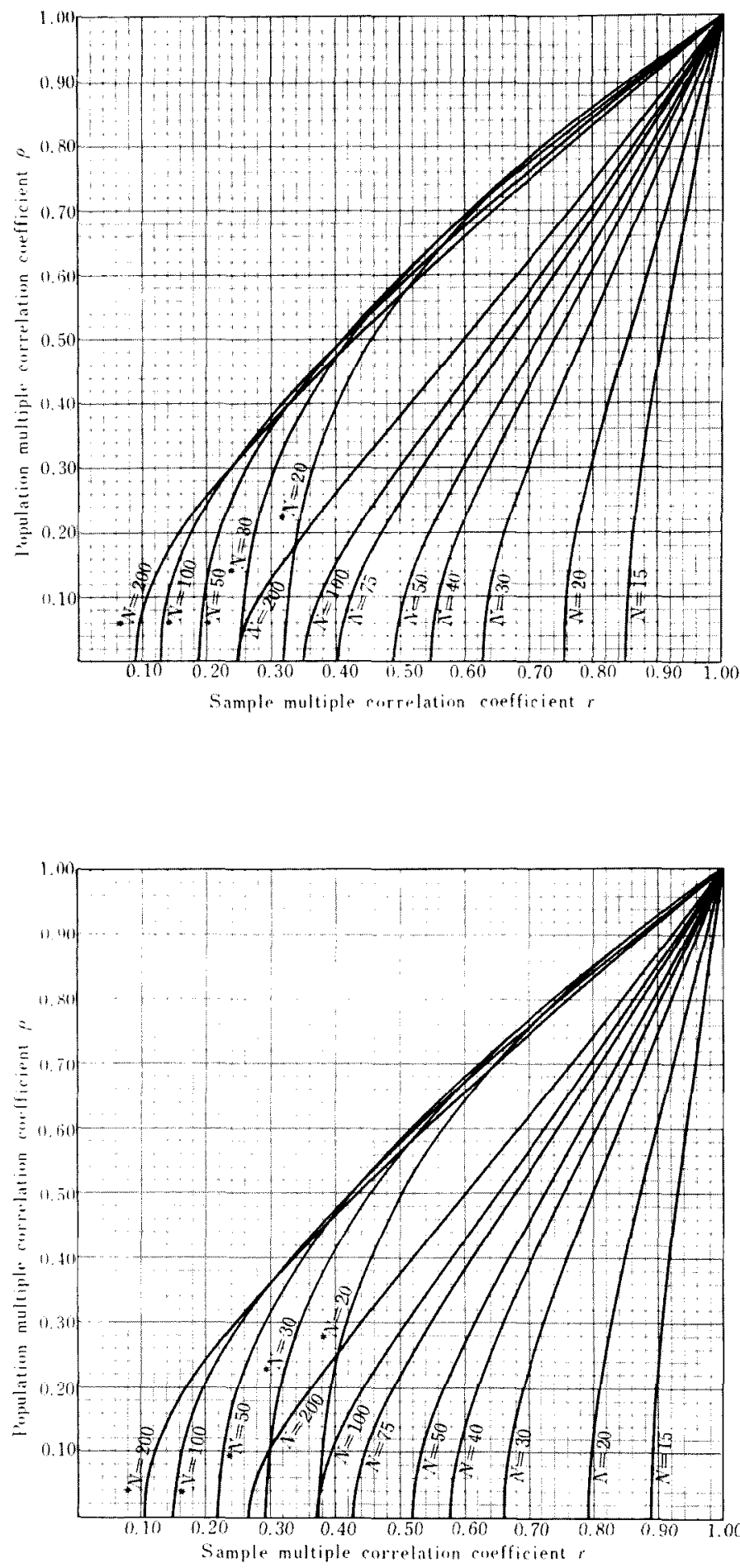

FIg. 5. Curves of left and right $5 \%$ tail points of distribution of sample multiple correlation coefficient for the different sample sizes with 6 independent variables. (* Curves for the lower tails)
FIG. 6. Curves of left and right $5 \%$ tail points of distribution of sample multiple corrclation coefficient for the different sample sizes with 7 independent variables. (* Curves for the lower tails) 
Fra. 7. Curves of left and right $5^{\circ}$ tail points of distribution of sample multiple correlation coefficient for the different sample sizes with 8 independent variables. (* Curves for the lower tails)
Fig. 8. Curves of left and right $5 \%$ tail points of distribution of sample multiple correlation coefficient for the different sample sizes with 10 independent variables. (* Curves for the lower tails)
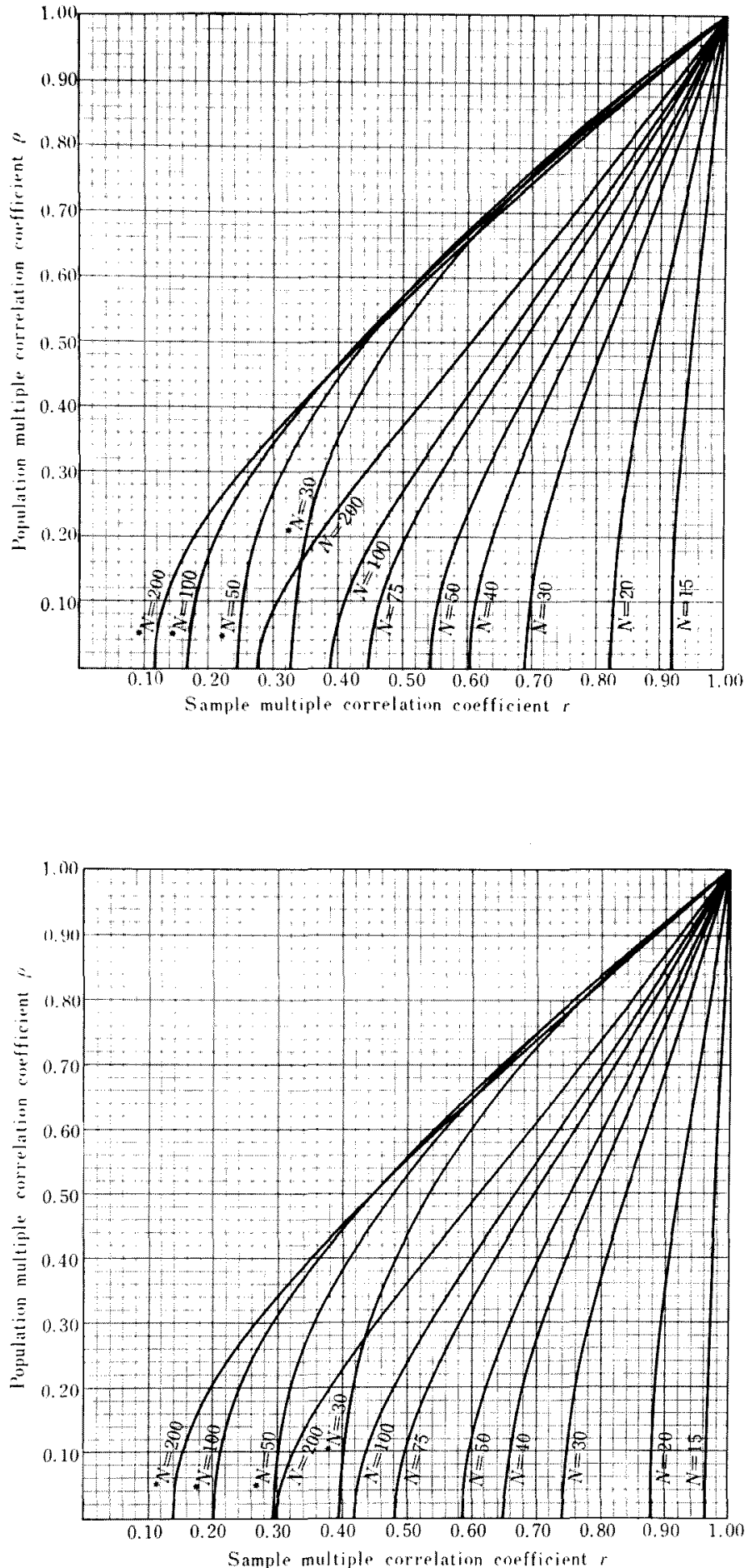
intersects the curves for $\mathcal{N}=100$ at $\rho_{U} \fallingdotseq 0.60$ and $\rho_{L} \doteqdot 0.34$, which are upper and lower limits for 90 percent confidence interval. These figures are also used for testing hypothesis $\mathrm{H}_{0}$ : $\rho=\rho_{0}$, where $\rho_{0}$ is not necessarily zero.

\section{REFERENCES}

ANDERson, T.W. 1958 An introduction to multivariate statistical analysis. New York: John Wiley.

Ezekiel, M., \& Fox, K.A. 1963 Methods of correlation and regression analysis. New York: John Wiley.
Fisher, R. A. 1928 The general sampling distribution of the multiple correlation coefficient. Proceedings of Royal Society. A, 121, 654-673.

KRAMER, K. H. 1963 Tables for constructing confidence limits on the multiple correlation coefficient. Fournal of the American Statistical Association, 58, 1082-1085.

NABEYA, S. 1948 On the sampling distribution of a multiple correlation coefficient. Research Memorandum of Institute of Statistical Mathematics, Tokyo, 4, 381-385 (in Japanese).

Yamauchi, J. (Ed.) 1972 Statistical tables and formulas with computer applicalions. Japanese Standards Association.

(Received Nov. 2, 1973) 